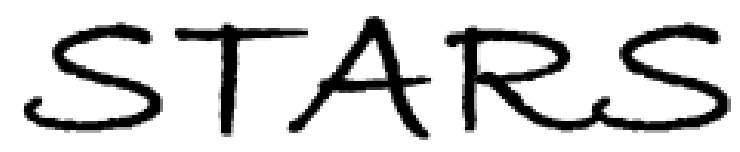

University of Central Florida

STARS

$1-1-2005$

\title{
Evoking affordances in virtual environments via sensory-stimuli substitution
}

David C. Gross

Kay M. Stanney

University of Central Florida

LT. Joseph Cohn

Find similar works at: https://stars.library.ucf.edu/facultybib2000

University of Central Florida Libraries http://library.ucf.edu

This Article is brought to you for free and open access by the Faculty Bibliography at STARS. It has been accepted for inclusion in Faculty Bibliography 2000s by an authorized administrator of STARS. For more information, please contactSTARS@ucf.edu.

\section{Recommended Citation}

Gross, David C.; Stanney, Kay M.; and Cohn, LT. Joseph, "Evoking affordances in virtual environments via sensory-stimuli substitution" (2005). Faculty Bibliography 2000s. 5231.

https://stars.library.ucf.edu/facultybib2000/5231

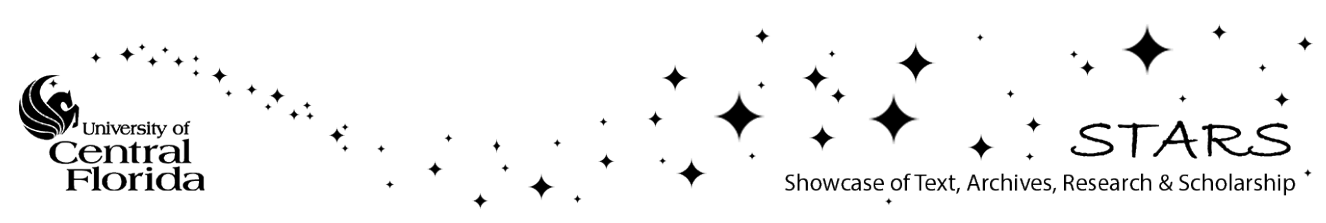




\section{Evoking Affordances in Virtual Environments via Sensory-Stimuli Substitution}

\begin{abstract}
Artifacts in an environment afford to their observers utility and function directly through specific object characteristics (e.g., mediums, surfaces, substances). Virtual environments (VEs) similarly seek to afford specific utility to their users, whether it is for training, education, or entertainment, thus it seems natural to consider basing VE designs on affordances. Such affordance-based design should yield significant benefits by providing designs that behave in more understandable and intuitive manners. These designs should be easier to learn, adapt better to user tasks, and frustrate users less by allowing use of the same skills acquired via realworld interactions. In order to realize affordance-based VE designs, the types of appropriate affordances and means of realizing these affordances must be identified. Currently, however, affordances lack a theoretical and operational basis for such application. The present paper suggests functions that affordances should support and provides a conceptual model for realizing affordances based on sensorystimuli-substitution schemes.
\end{abstract}

\section{Introduction}

A core human-performance issue in VEs is determining how to design the objects to be represented in the virtual environment. If designed effectively, VEs should allow users to readily perceive what can and should be done in the virtual world. This suggests that a productive area of research is the extension and application of theories of human perception to the design of VEs (Pratt, Zyda, \& Kelleher, 1995; Barnard \& May, 1999). The issue for VE designers is inverted, however, from that addressed by traditional psychological theories. Instead of understanding how humans perceive what naturally occurs in the real world, VE designers

Presence, Vol. 14, No. 4, August 2005, 482-491

(๑) 2005 by the Massachusetts Institute of Technology need to understand how their designs support and exploit natural perception within the virtual world they represent. More specifically, the issue is how users perceive the objects, properties, and behaviors selected by VE designers for inclusion in the virtual world; it is through the effective (or ineffectual) design of these artifacts that designers communicate with their users (Anders, 1999). Beyond physical artifacts, designers must also consider semantic, cultural, and logical relations in their designs. All these factors can drive and constrain how an artifact is perceived (Norman, 1988).

The theory of direct perception asserts that humans can (and do) directly perceive the possible actions in an environment, or conversely what the environment affords to the human (Gordon, 1989). Yet how VE designs might evoke specific affordances, and thus how the theory of affordances should influence VE design, is not a straightforward matter. Many researchers feel the pursuit would be fruitful (Norman, 1988; Vicente \& Rasmussen, 1992; Eberts, 1994; Pejtersen \& Rasmussen, 1997; St. Amant, 1999; Lintern, 2000 ). However, Biocca (2001) noted that the bulk of affordance-related research in human-computer interface design has not actually applied or developed the theory of direct perception, but instead has interpreted affordances as a metaphor weakly linked to Gibson's (1979) original premises. There is a limited amount of research that meets Biocca's implicit test. One example is St. Amant,

\section{David C. Gross}

The Boeing Company

Huntsville, AL 35824 USA

david.c.gross@boeing.com

Kay M. Stanney

Industrial Engineering \& Management Systems Department University of Central Florida

Orlando, FL 32816 USA

\section{Lt. Joseph Cohn}

Naval Air Warfare Center Training Systems Division

Orlando, FL 32826 USA 
which focused on the straightforward "real" affordances presented by the WIMP (window-icon-menu-pointing device) interface; for example, the direct perception afforded by the mouse's "click." This approach is dissatisfying for the design of immersive virtual environments because the issue in such interfaces is not the perceptions evoked by interface metaphors, but the perceptions evoked by represented objects in the virtual environment, and the design objective of making those evoked perceptions similar to the real world the virtual environment is intended to emulate.

A second example is ecological interface design (EID), a theoretical framework for interface design (Vicente \& Rasmussen, 1992). It is based on two seminal concepts from cognitive engineering research, the abstraction hierarchy (AH) and the skills, rules, knowledge (SRK) framework. The AH is a multilevel knowledge-representation framework that can be used to develop physical and functional work-domain models, as well as the mappings between them. It is used in EID to identify the information content and structure of the interface. The SRK framework defines three qualitatively different ways in which people can process information. At the skillbased level, behavior is governed by a dynamic world model that allows people to engage in fluid perceptualmotor control; at the rule-based level, behavior is governed by rules that directly map perceptual cues in the environment to appropriate actions, without any mediating processing; finally, at the knowledge-based level, behavior is governed by a symbolic mental model that allows people to engage in analytical problem solving. The SRK framework is used in EID to identify how information should be displayed in an interface. The idea is to take advantage of operators' powerful pattern recognition and psychomotor abilities, allowing people to deploy everyday skills that have been honed through evolution. Thus, EID recommends that information be presented in such a way as to promote skill- and rulebased behavior, allowing operators to deal with task demands in a relatively efficient and reliable manner.

Knowledge-based behavior is also supported by embedding an $\mathrm{AH}$ representation of the work domain in the interface. This provides operators with an external visualization of system structure and dynamics, which offers support during novel situations requiring adaptive problem solving (Vicente, 1999). This approach is somewhat dissatisfying for the design of immersive virtual environments because it depends on a domain model of the work to be performed, whereas the design objectives for some immersive environments do not include an a priori definition of specific work to be performed ("freeplay" environments). Also, the SRK framework leverages the user's cognitive abilities such as pattern recognition, but it does not extend as well to tasks that depend on a range of sensory modalities, such as walking. Nor is it as helpful in environments lacking sensory modalities, such as is the case in present virtual environments, as it is in more general environments.

The present work suggests a new approach to satisfying the design objective of evoking affordances in the virtual environment corresponding to those of the real world being modeled, by applying direct perception to the design of immersive interfaces based on sensorystimuli-substitution schemes for sensory modalities that are missing or absent in virtual environments.

Direct perception has a theoretical shortcoming for perception in synthetic environments such as virtual worlds. Direct perception specifically addresses perception in a natural environment (which is why the theory is sometimes called ecological perception), and denies that synthetic environments are intrinsically ecological valid (Gibson, 1979). The ecological invalidity of synthetic environments has been previously empirically confirmed (e.g., Stappers, 1999).

Direct perception will operate on pictures and other synthetic environments, but there is no assurance that the perceptions will necessarily correspond to those evoked by the real world that the synthetic environment models, which is the design objective for many immersive virtual environments. Direct perception also offers no method for applying the theory to interactive system design. Okamoto (1997) stated that affordance theory does not seem to be practical because the theory is so philosophical that designers have no idea how to apply theoretical statements to the design of human interfaces. Eberts (1994) suggested that deciding which affordance to associate with a design is an extremely difficult problem. The lack of a rigorous theoretical 
foundation for affordances means there is presently no practical technique for discovering affordances (Fitzgerald \& Goldstein, 1997). Bingham and Muchisky (1991) argued that while some researchers have suggested that affordances are revealed in the intrinsic properties of an item, others have attempted to find an affordance in the dynamic behavior of the item. Neither approach has proven generally effective. Indeed, Gibson (1979) claimed that affordances are facts about the animalenvironment system, facts that exist whether or not they are perceived or detected, facts whose existence and importance are entirely independent of the person's knowledge, perception, and so on. This contrasts with Norman's (1988) interpretation of the concept of affordances to refer to a perceiver's understanding of possibilities for action.

It may be that affordances can be identified only when bound to the context in which they are used. For example, Gleaves (1998) identified affordances associated with textbooks and then translated them into design principles for hypermedia. The first of these affordances was riffleability (i.e., the ability to leaf through hastily), which led to the suggestion that hypermedia designs should provide a visual index to content. The second was comparability (i.e., comparison of noncontiguous information), which led to the suggestion to provide multiple views of content. Bookmarkability allows for direct access to desired content. Annotatability allows for the ability to readily make notations. Highlightability allows for the ability to select and underscore content. Excerptability allows for the extraction of content. Progress visibility allows for identification of advancement through content. Finally, closure provides bounds on content, allowing users to build confidence and offering a source of orientation within the content. Taken together, the key functions provided by these affordances are to allow users to:

- orient

- identify

- select

- access

- organize, and

- integrate.
Table I. Affordance Realization Dependencies

Affordances depend on objects in the environment.

Affordances depend on the organism's action capabilities.

Affordances depend on physical characteristics of the observer.

Affordances depend on the organism's sources of sensory stimuli.

Affordances depend on integration of multimodal sensory stimuli.

Affordances arise as the organism learns to act within its environment.

Affordances for VEs should likely provide these same functions. But how will designs enable the realization of such affordances?

\section{Realization of Affordances}

Our synthesis of the affordance literature (Gross, Stanney, \& Cohn, 2001) has led to a new approach for making the theory of direct perception an operational technique for the design of virtual environments. This approach is based on six assertions about how the various factors involved in perception are integrated into a percept via the realization of an affordance (see Table 1). These assertions lead to the premise that affordances are realized through the integration of environmental and state stimuli, which in turn interact with the experience gained through decision making, which in turn affects an individual's knowledge of internal state, action capabilities, and body stature in an environment. This premise is captured in the conceptual model presented in Figure 1.

Designed environments, such as VEs, are not intrinsically ecologically valid, and so may not support the realization of affordances as desired by their designers. Since they do represent an environment of sorts, they will support realization of some affordances, just not necessarily those desired, especially not those of the reality that the synthetic environment may be attempting to represent. While researchers have discussed the prob- 


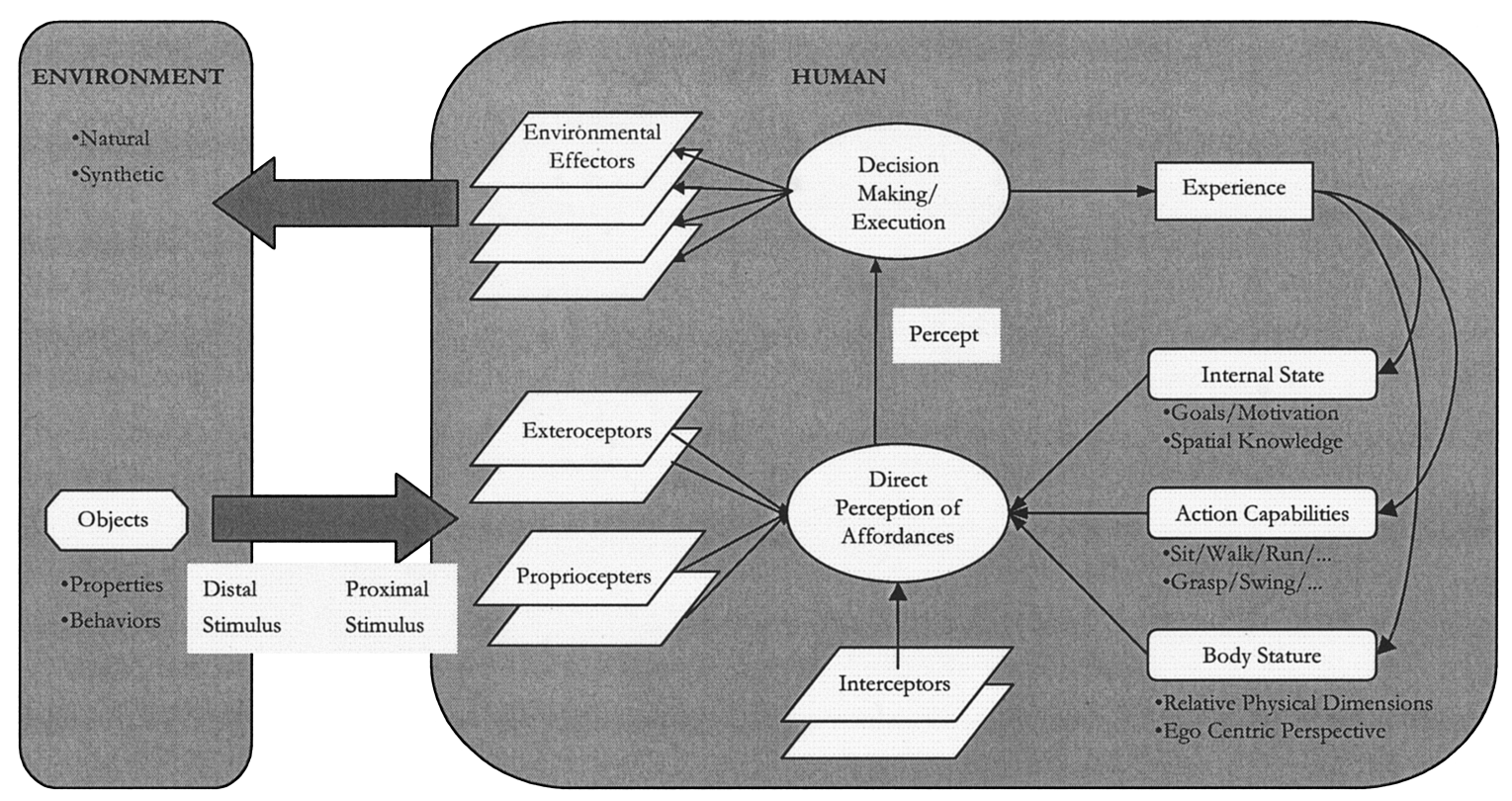

Figure I. Realization and exploitation of affordances.

lem of ecological invalidity in general terms (Kennedy, 1974; Gibson, 1979; Norman, 1988; Fitzgerald \& Goldstein, 1997), Figure 1 presents a context for decomposing the causes of such invalidity and thus can potentially guide VE designers in generating more ecologically valid designs.

The most basic source of affordance-realization failures is an inaccurate or insufficient sensory stimulus, that is, below the modality's response threshold (see Figure 1). Rose, Jankowski, and Senior (1997) showed that infants are capable of recognizing line drawings missing much of their contour. Yet the infants failed to discriminate between two extremely impoverished figures (more than $66 \%$ of their contours missing) because they provided an inaccurate sensory stimulus. In terms of Figure 1, this would be a failure of proximal stimuli to activate the sensory system. Thus, to realize the perception of affordances, stimuli must be above the appropriate sensory-modality threshold. By sensory-modality threshold, we mean the signal strength of stimuli that is sufficient to activate a particular sensory modality, for example, a sound loud enough to be perceived.

The assertion that stimuli must be above the appropriate sensory-modality threshold to enable the realization of an affordance is somewhat surprising against the context of Gibson's (1979) theory of direct perception. For example, "There is no threshold for information comparable to a stimulus threshold" (p. 243). So, how are we reconciling our first condition with Gibson's? First, Gibson is not arguing that stimulus thresholds do not exist, simply that information is different than stimulation. Our position is that stimulation is necessary but not sufficient for information to arise within the organism-there are a number of additional factors necessary for information to arise, which the following discussion addresses.

Another source of realization failure especially pertinent to VEs is the absence or inaccuracy of required multimodal sensory stimulation to realize an affordance. Warren and Whang (1987) found in their study of passability that when approaching a doorway whose ratio of width to shoulder width was less than $1: 1.3$, subjects would rotate their body. This is an indication of the kinesthetic and visual sense interacting with the action of walking when perceiving the affordance of passability. Wertheim (1994) argued that the vection created with an optokinetic drum must involve extra retinal signals, namely vestibular afferents. This argument led Wertheim to argue more generally that visual-vestibular interaction is crucial for correct perception in ecologically 
valid environments. Oudejans, Michaels, Bakker, and Dolne (1996) presented a study on the catchability of fly balls, namely the ability of subjects to judge whether they could catch balls thrown at various heights in the sagittal plane, that is, thrown straight at the subject. This study demonstrated that perceiving the affordance of catchability depends on kinematics of the subject's body as well as the movement of the ball by showing that the ratio of balls judged catchable to those actually catchable varied with the dynamic information available to the observer. Taken together, this research suggests that correct realization of affordances may be perceived cross-modally, in particular crossing between the visual and other sensory systems. This poses a problem for VE design because even though stimulation of propriocepters and interceptors is required for realization of some affordances, there exist few reliable and economical means of providing these cues via artificial stimuli (Stuart, 1996). Indeed, instead of receiving artificial stimulation correlated with the VE, users experience continual and generally contradictory stimulation from the natural environment. In terms of Figure 1, this would be a breakdown of the interfaces between propriocepters and interceptors and direct perception. Thus, to correctly perceive multimodal affordances, propriocepters and interceptors must be correctly activated or appropriate sensory-substitution schemes must be identified. Indeed, we believe that correlation of multimodal stimulation is a key mechanism in how information arises within the context of Gibson's theory of direction perception.

Another source of realization failure may be inadequate perception of body stature in the environment. Gibson (1979) suggested that environmental properties have to be measured relative to the body, without specifying how this relationship is defined. Warren (1984) argued that this is a visual perception; however, he acknowledges that what must be occurring is perception of environmental properties relative to the observer's capabilities. He proposed that the critical (maximum) and optimal values of an environmental property, relevant to performing an action, are an invariant proportion of some aspect of the actor's body scale. He demonstrated this by showing that the climbing affordance on stairs is influenced by visual information about the height of the step and internal-state information about the length of the observer's leg. Marik (1987) extended Warren's finding by demonstrating that the critical boundaries for the affordances of sitting and stair climbing are scaled with reference to the actor's eye height. Warren and Whang (1987) found that perception of the passability affordance depends on both visual information about aperture width and internal knowledge of shoulder width. At shoulder widths to doorway ratios of less than 1:1.3, the subjects perceived insufficient clearance, and rotated their bodies to proceed through the opening. These findings are significant for understanding how affordances may be perceived in VEs, because they indicate that information about body stature in the environment is required if the user is to correctly realize affordances. Since VE designs frequently do not provide users with information about their own stature (Anders, 1999), this may impede the realization of affordances. In terms of Figure 1, this would be a loss of the information represented by "stature" in the environment block.

Realization failures associated with designed environments such as VEs may also occur due to inadequate perception of action capabilities in the environment (see Figure 1, action-capabilities block). For example, VEs often do not match the real world in terms of what can be done. Indeed, providing different action capabilities is one of the motivations for using virtual environments. Although VEs can evoke an immersive experience, this does not lead automatically to a natural interface. To experience a natural interface, the user should be able to perceive what can be done via the interface.

Since VEs represent a kind of reality and seek to immerse their users in that reality, users have a reasonable expectation that the environment behaves like reality or in easily understandable deviations from reality. St. Amant (1999) argued that:

Users only rarely encounter problems in using specific widgets, and remedies at the given level of abstraction can only provide a limited benefit. Problems more often arise at a conceptual level. What is it possible to do in the interface? Why can't a given operator be 
executed in the current state? How can one reach a desired goal? (p. 342)

Virtual-environment interface designs define what users are capable of doing (e.g., moving, orienting), thereby defining the context for and level of realism of affordances included in the environment. In the example of the passability-affordance study (Warren \& Whang, 1987), it is not clear that a VE user would know that body rotation is possible in any particular interface, much less necessary or helpful to gain passage. In the example of the catchability study (Oudejans et al., 1996), it is possible that VE users may not know how to maneuver so as to catch something if locomotion is represented fundamentally different than in the natural environment. As a result, in such environments, due to a lack of cues (e.g., body stature, propriocepters, interceptors) users may not be able to depend on their experience-based expectations about manipulating their natural environment to realize such affordances. In such circumstances, it may be possible to support affordance realization via sensory-substitution schemes.

\section{Sensory Substitution via Affordance- Based Designs}

The potential breakdowns in the realization of affordances in VEs can potentially be overcome by (a) correlating sensory stimuli to the experience represented in the virtual world, (b) determining that a particular modality is irrelevant to the experience, or (c) substituting information via another modality for missing modalities. The latter approach, sensory-substitution schemes, may have the greatest potential as it is not limited by technology (as is the first approach) and does not require diminishment of an experience (as does the second approach). Sensory-substitution schemes could potentially be used to replace missing stimuli required to evoke the realization of affordances.

There is evidence that sensory-substitution schemes can lead to correct perceptions, even though the current state of multimodal modeling in VE design is rudimentary (Popescu, Burdea, \& Trefftz, 2002). First, consider that all perception, not just complex reactions to an environment, is multimodal (Storms, 2002). Bregman (1990) believes that the auditory and visual modalities interact in order to specify the nature of certain events within a perceiver's environment. Eimer (2001) asserts that cross-modal links exist in spatial attention between vision, audition, and touch, and that the emerging evidence is that cross-modal links in spatial attention affect sensory-perception stages, but have less impact on later postperceptual stages. The body of evidence in the literature clearly indicates that under certain conditions, auditory-visual perceptual phenomena do exist (Storms). The implication for the present work is that since sensory modalities are complementary, they may be to some extent redundant and therefore replaceable by stimuli in other modalities.

Next, consider the evidence that there may be underlying neurological factors that determine perception more than the specific sensory modalities involved. Specifically, if the brain is structured to favor perceptions arising from multimodal stimuli, then correct perception may depend on multimodal stimuli. Stein and Meredith (1993) found neurological evidence for this position, based primarily on their study of cats. They conclude:

The spatial register among the receptive fields of multisensory neurons and their temporal response properties provide a neural substrate for enhancing responses to stimuli that covary in space and time and for degrading responses that are not spatially and temporally related. (p. 172)

The implication to the present work is that since brain structure may be favorable toward perception of multimodal stimuli, then in environments such as VEs, where specific sensory modalities are impoverished, correct perception may require stimuli that substitute for the improvised modality.

Next, consider that there is evidence that stimulation in one modality can complement or distract from stimulation in other modalities. Curran, Schacter, and Galluccio (1999) provided evidence for cross-modal priming, specifically that verbal data primed the perception of subsequent visual images. Henneman and Long (1954) indicated that there has been very little experimental 
evidence comparing audition and vision as channels for data presentation, and concluded that most auditoryvisual intersensory studies have focused on sensory thresholds as opposed to suprathreshold levels that typify actual perceptual phenomena. Rode, Salkovskis, and Jack (2001) showed that an audio distraction significantly lowered perceived pain, as measured by subjective report and improvement on a muscle-stamina task. Spence and Driver (1997) argued that people can monitor cross-modal stimuli as effectively as a single stimulus. The implication for the present work is that the ability for stimuli in some modalities to distract from stimuli in other modalities suggests that cross-modal sensorysubstitution schemes can be an effective VE design approach, within yet-to-be-determined limits.

Finally, Shimojo and Shams (2001) noted that the direction of cross-modal interactions was previously thought to be determined by the relative appropriateness of the modalities involved in a task. Emerging evidence, however, is that the direction depends at least in part on the structural (spatial versus temporal) nature of the stimuli. They, along with other researchers, such as Monder and Amirault (1998), have noted the strong association between spatial perceptions and visual stimuli, and temporal perceptions and auditory stimuli. Shimojo and Shams suggested that the brain may accept stimuli in a modality other than the natural mode, if the underlying spatial-temporal structure of the stimuli is retained. This is similar to the argument in favor of stimulus-response compatibility advanced in, for example, Eberts (1994). In general, relationships between stimuli and responses are compatible when they facilitate action. The example cited in Eberts is for a stovetop design, in which the controls are laid out in the same pattern as the stove burners. The implication for the present work is that substitution schemes should match stimuli to the spatial-temporal nature of the perception.

The foregoing discussion presents the general case that substitution schemes may succeed; however, the specific form of the substitution scheme has not been considered. There are many different conceivable sensory-substitution schemes; the literature provides some guidance on which are likely to succeed. First, consider that the only mechanism that a VE designer has for communicating with its users is through the properties of objects represented in the virtual world. Therefore, the only possible sensory-substitution schemes will be ones that exploit the exterocepter sensory systems (i.e. vision, audition) and propriocepters (i.e., cutaneous [or haptic], gustation, and olfaction).

The most obvious scheme would be to provide visual cues to substitute for the missing sensory stimulation. This is because: (a) visual stimuli in VEs are already available and powerful, (b) the visual sensory system has the broadest band input to the brain (Sharma, Pavlovic, \& Huang, 1998), and (c) the visual-dominance effect often occurs (Storms, 2002).

The visual sense is generally considered the dominant sense. Unless there are significant differences in the intensities of information gathered via different modalities, visual stimuli have been found to have a greater influence on perception via other modalities, as compared to the influence of other modalities on the visual sense (Stein \& Meredith, 1993). Wickens (1992) explained visual dominance by stating: "If visual stimuli are appearing at the same frequency and providing information of the same general type or importance as auditory or propriocepter stimuli, biases toward the visual source at the expense of the other two (auditory and propriocepter) will be expected" (p. 108). Cohn, DiZio, and Lackner (2000) presented an example of how a visually pure environment can elicit a visual reflex rather than a straightforward perception of the sensory data. More specifically, they showed that visual information about body motion alone is sufficient to elicit directionally appropriate Coriolis compensations. Srinivasan, Beauregard, and Brock (1996) demonstrated the effect of visual dominance on haptic perception. In this experiment, participants had to discriminate the stiffness of two virtual springs when provided with independent visual and haptic feedback about their stiffness. When the visual stiffness stimuli conflicted with the haptic stiffness stimuli, participants judged stiffness consistent with the visual stimuli in preference to the haptic feedback. Ivanenko, Viaud-Delmon, Sigler, Israel, and Berthoz (1998) showed that humans exposed to a VE undergo adaptations at the sensory level, including adaptations of the vestibulo-ocular reflex and angular dis- 
placement perception, and that these adaptations may occur independently of one another. Prothero et al. (1999) were able to normalize the ill effects associated with missing vestibular cues through the provision of a visual stimulus calibrated to the stationary stance of observers immersed in a virtual world. Taken together, these studies provide evidence that visual stimuli can evoke a reaction in other sensory systems. Thus, visual stimuli alone may evoke a perception comparable to that evoked by multimodal stimuli in the natural environment.

Vision is not, however, the dominant sense in every circumstance. Substitution schemes other than purely visual stimuli should thus be considered. During signal detection, which is temporal in nature and typically associated with sustained attention or vigilance, the auditory channel proves dominant over the visual channel, which is why warning signals are typically produced with auditory devices (Storms, 2002). There is also evidence that the intensity of visual images can be enhanced by audio stimuli (Shimojo \& Shams, 2001).

What about sensory-substitution schemes exploiting propriocepters? There is very little research into providing sensory stimulation for gustation and olfaction, but somewhat more for haptic stimulation. Proffitt and Kaiser (1995) provided an example of haptic dominance. They asked participants to estimate the incline of a hill while provided with audio, visual, and haptic stimuli, and the most accurate estimates resulted from the haptic interaction. In general, however, there is little evidence encouraging approaches exploiting propriocepters (Popescu et al., 2002). Therefore, initially, sensory-substitution schemes may be based on exterocepters, that is, visual and auditory, instead of propriocepters stimuli.

Several authors have suggested that sensory substitutions should map spatial information to visual substitution cues and temporal information to auditory cues (Popescu et al., 2002; Shimojo \& Shams, 2001). This suggests that the structure of the information to be perceived might indicate the best substitution scheme.

The success of a sensory-substitution scheme depends on an understanding of how several factors are integrated into a percept, including multimodal stimuli, available effectors, experience, attention, consistency,
Table 2. Design Recommendations for Sensory Substitutions to Evoke Affordances

\begin{tabular}{lc}
\hline Condition & Design Guideline \\
\hline $\begin{array}{c}\text { Where the affordance is } \\
\text { perceived in the }\end{array}$ & $\begin{array}{c}\text { No substitutional stimuli } \\
\text { should be required } \\
\text { natural environment } \\
\text { with purely visual or }\end{array}$ \\
auditory stimuli & \\
Where the affordance is & Substitute for missing \\
essentially a spatial & sensory stimuli with \\
perception & visual stimuli \\
Where the affordance is & Substitute for missing \\
essentially a temporal & sensory stimuli with \\
perception & auditory stimuli
\end{tabular}

motivation, organization, attitude, and learning (see Figure 1). If this integration process is well understood, then designers can exploit that understanding in their design of sensory-substitution schemes. The foregoing discussion led to a number of design recommendations for implementing sensory-substitution schemes for missing sensory modalities, which should evoke specific affordances (see Table 2).

\section{Conclusions}

The utility of affordance-based design is that it should allow users to readily perceive what can and should be done in a virtual environment. Yet a given virtual environment offers its users the affordances intended by the designer only if the design enables their realization. The present work argues that realization of affordances requires sufficiency in a number of related factors: sufficient sensory stimulation, including multimodal sensory simulation, sufficient perception on body stature, and sufficient perception of action capabilities, all of which are challenging to provide with present-day VE technology. The work presented a conceptual model for designing appropriate sensory-substitution schemes within the constraints of existing VE technology that can support the realization of specific affordances. This 
work was followed by an experimental program empirically validating the conceptual mode, and the guidelines for substitution schemes as presented in Table 2. The results of that experimental program are being prepared for publication.

\section{References}

Anders, P. (1999). Envisioning cyberspace: Designing 3D electronic spaces. New York: McGraw-Hill.

Barnard, P. J., \& May, J. (1999). Representing cognitive activity in complex tasks. Human-Computer Interaction, 14, 93-159.

Bingham, G., \& Muchisky, M. (1991). Affordances and dynamics: Graspability and center of mass perception (Tech. Rep. No. 60). Bloomington, IN: Indiana University, Program of Cognitive Science.

Biocca, F. (2001). Inserting the presence of mind into a philosophy of presence: A response to Sheridan and Moantovani and Riva. Presence: Teleoperators and Virtual Environments, 10(5), 546-556.

Bregman, A. S. (1990). Auditory scene analysis. Cambridge, MA: MIT Press.

Cohn, J. V. DiZio, P., \& Lackner J. R. (2000). Reaching during virtual rotation: Context specific compensations for expected Coriolis effects. Journal of Neurophysiology, 83(6), 3230-3240.

Curran, T., Schacter, D. L., \& Galluccio, L. (1999). Crossmodal priming and explicit memory in patients with verbal production deficits. Brain and Cognition, 29, 133-146.

Eberts, R. (1994). User interface design. Englewood Cliffs, NJ: Prentice Hall.

Eimer, M. (2001). Crossmodal links in spatial attention between vision, audition, and touch: Evidence from eventrelated brain potentials. Neuropsychologia, 29, 1292-1303.

Fitzgerald, W., \& Goldstein, E. (1997). Honesty of affordance, a white paper. Evanston, IL: Neodesic Corporation.

Gibson, J. J. (1979). The ecological approach to visual perception. Hillsdale, NJ: Erbaum.

Gleaves, R. (1998). Learning from books: Eight design principles for instructional hypermedia. Available from: http:// et.sdsu.edu/rgleaves/ET640.htm.

Gordon, I. E. (1989). Theories of visual perception. Chichester, UK: Wiley.

Gross, D., Stanney, K., \& Cohn, J. (2001). Toward a theory of affordance-based design of virtual environments. HCIInternational 2001.

Henneman, R. H., \& Long, E. R. (1954). A comparison of the visual and auditory senses as channels for data presentation (Tech. Rep. No. 54-363). Dayton, OH: USAF, Wright Air Development Center.

Ivanenko, Y. P., Viaud-Delmon, I., Sigler, I., Israel, I., \& Berthoz, A. (1998). The vestibulo-ocular reflex and angular displacement perception in darkness in humans: Adaptation to a virtual environment. Neuroscience Letters, 241, 167170.

Kennedy, J. M. (1974). Perception, pictures, and the etcetera principle. In R. B. MacLeod \& H. L. Pick, Jr. (Eds.), Perception: Essays in honor of James J. Gibson (pp. 209-226). London: Cornell University Press.

Lintern, G. (2000). An affordance-based perspective on human-machine interface design. Ecological Psychology, 12(1), 65-69.

Marik, L. S. (1987). Eyeheight-scaled information about affordances: A study of sitting and stair climbing. Journal of Experimental Psychology: Human Perception and Performance, 13(3), 361-370.

Monder, T. A., \& Amirault, K. J. (1998). Effect of same- and different-modality spatial cues on auditory and visual target identification. Journal of Experimental Psychology: Human Perception and Performance, 24(3), 745-755.

Norman, D. A. (1988). The design of everyday things. New York: Doubleday.

Okamoto, A. (1997). Toward rehabilitation cognitive engineering-Gap between theory and practice in the human interface of information processing devices for people with disabilities. In M. L. Smith, G. Salvendy, \& R. J. Koubek (Eds.), Design of computing systems: Social and ergonomics considerations (pp. 551-554). New York: Elsevier Science.

Oudejans, R. R. D., Michaels, C. F., Bakker, F. C., \& Dolne, M. A. (1996). The relevance of action in perceiving affordances: Perception of catchableness of fly balls. Journal of Experimental Psychology: Human Perception and Performance, 22(4), 879-892.

Pejtersen, A. M., \& Rasmussen, J. (1997). Ecological information systems and support of learning: Coupling work domain information to user characteristics. In M. Helander, T. K. Landauer, \& P. V. Prabhu (Eds.), Handbook of human-computer interaction (2nd ed. pp. 49-63). Amsterdam: North-Holland.

Popescu, G. V., Burdea, G. C., \& Trefftz, H. (2002). Multimodal interaction modeling. In K. M. Stanney (Ed.), 
Handbook of virtual environments: Design implementation and application (pp. 435-454). Mahwah, NJ: Erlbaum.

Pratt, D. R., Zyda, M., \& Kelleher, K. (1995). Virtual reality in the mind of the beholder. Computer, 17-26.

Proffitt, D. R., \& Kaiser, M. K. (1995, March). Human factors in virtual reality development. Tutorial at the Virtual Reality Annual International Symposium, Research Triangle, NC.

Prothero, J. D., Draper, M. H., Furness, T. A., Parker, D. E., \& Wells, M. J. (1999). The use of an independent visual background to reduce simulator side-effects. Journal of Aviation, Space, and Environmental Medicine, 70(3), 277-283.

Rode, S., Salkovskis, P. M., \& Jack, T. (2001). An experimental study of attention, labeling, and memory in people suffering from chronic pain. Pain, 94, 193-203.

Rose, S. A., Jankowski, J. J., \& Senior, G. J. (1997). Infants' recognition of contour-deleted figures. Journal of Experimental Psychology: Human Perception and Performance, 23(4), 1206-1217.

Sharma, R., Pavlovic, V. I., \& Huang, T. S. (1998). Toward multimodal human-computer interface. Proceedings of the IEEE, 86(5), 853-869.

Shimojo, S., \& Shams, L. (2001). Sensory modalities are not separate modalities: Plasticity and interactions. Current Opinions in Neurobiology, 11, 505-509.

Spence, C., \& Driver, J. (1997). Audiovisual links in exogenous covert spatial orienting. Perception and Psychophysics, $59(1), 1-22$.

Srinivasan, M. A., Beauregard, G. L., \& Brock, D. L. (1996). The impact of visual information on the haptic perception of stiffness in virtual environments. Proceedings of the ASME Dynamics Systems and Control Division, 58, 555-559.

St. Amant, R. (1999). User interface affordances in a planning representation. Human-Computer Interaction, 14, 317355.

Stappers, P. J. (1999). Critical ratios as behavioral indices of presence. Second International Workshop on Presence, 6-7 April.

Stein, B. E., \& Meredith, M. A. (1993). The merging of the senses. Cambridge, MA: MIT Press.

Storms, R. L. (2002). Auditory-visual cross-modality interaction and illusions. In K. M. Stanney (Ed.), Handbook of virtual environments: Design implementation and application (pp. 455-470). Mahwah, NJ: Erlbaum.

Stuart, R. (1996). The design of virtual environments. New York: McGraw-Hill.

Vicente, K. (1999). Ecological interface design: Supporting operator adaptation, continuous learning, and distributed, collaborative work. Applied Cognitive Science and Human Centered Systems, 93-97.

Vicente, K., \& Rasmussen, J. (1992). Ecological interface design: Theoretical foundations. IEEE Transactions on Systems, Man, and Cybernetics, SMC-22, 589-606.

Warren, W. H., Jr. (1984). Perceiving affordances: Visual guidance of stair climbing. Journal of Experimental Psychology: Human Perception and Performance, 10(5), 683-703.

Warren, W. H., Jr., \& Whang, S. (1987). Visual guidance of walking through apertures: Body-scaled information for affordances. Journal of Experimental Psychology: Human Perception and Performance, 13(3), 371-383.

Wertheim, A. H. (1994). Motion perception during selfmotion: The direct versus inferential controversy revisited. Behavioral and Brain Sciences, 17(2), 293-355.

Wickens, C. D. (1992). Engineering psychology and human performance (2nd ed.). New York: Harper Collins. 